\title{
Sobre um Schizotrypanum dos morcegos Lonchoglossa ecaudata e Carollia perspicillata do Brasil *
}

\author{
pelo \\ Dr. Eimmanuel Dias \\ (Com 1 estampa)
}

\begin{abstract}
Em recente excursão á zona do Rio Paraíba compreendendo as estações de Benjamin Constant e de Teixeira Soares, em Minas Gerais, tornámos a examinar o sangue de numerosos morcegos, á cata de hemoparasitos. Já nessa região assinalaramos (Dias, 1936) a infecção por Schizotrypanum nas especies P'hyllostomus hastatus Pallas e Carollia perspicillata (L.). Fizemos, outrosim, pesquisas em morcegos do Distrito Federal, desta vês com resultados positivos, ao contrario dos anteriores.
\end{abstract}

\section{MORCEGOS EXAMINADOS}

A pesquisa de tripanosomas no sangue periférico, pelo exame a fresco, foi praticada em 87 exemplares das mencionadas estações de Minas Gerais, tendo sido em alguns casos tambem feito o exame em gotas espessas. Os quirópteros eram examinados vivos ou recentemente morlos, e pertenciam a 9 especies distintas, conforme determinação bondosamente feita pelo Dr. Carlos Vieira, do Museu Paulista, que examinou um grupo de especimens.

A relação abaixo mostra o numero de individuos de cada uma das especies examinadas, á exceção das tres primeiras cuja discriminação não foi possivel no local, tendo-se perdido varios exemplares para fim de classsificação.

* Recebido para publicação a 25 de Maio de 1940 e dado á publicidade em Setembro de 1940. 
LISTA DOS MORCEGOS EXAMINADOS EM B. CONSTANT E T. SOARES, EM MARCO DE 1940

Carollia perspicillata

Lonchoglossa ecaudata

Glossophaga soricina

Phyllostomus hastatus

Positivos

Negativos

Vampirops lineatus

Artibeus jamaicensis

Chrotopterus auritus

Molossus rufus

Myotis nigricans

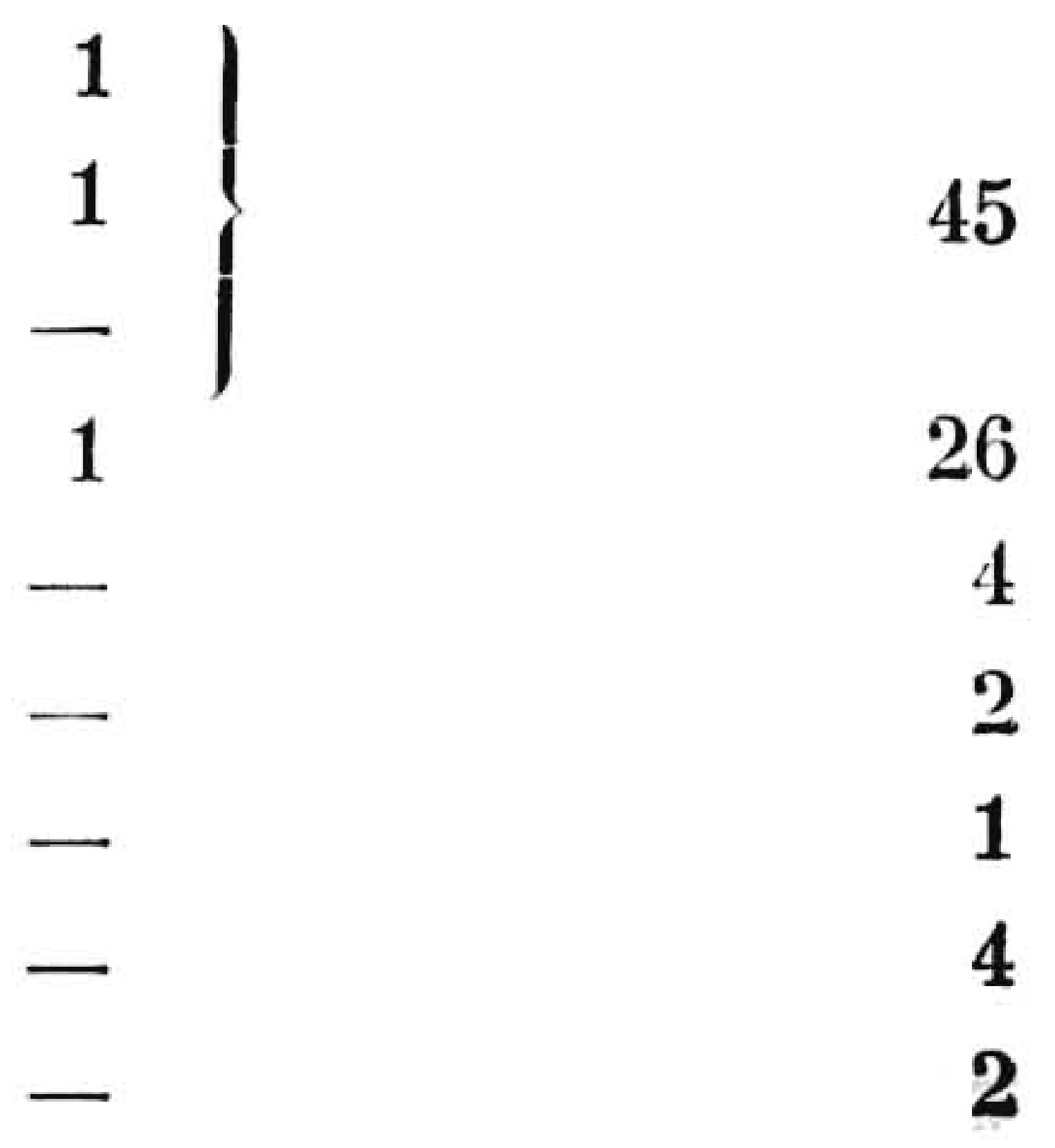

No Distrito Federal, capturámos na Estrada da Tijuca alguns Phyllostomideos das especies $C$. perspicillata, L. ecaudata e G. soricina.

\section{MORCEGOS INFECTADOS}

Dentre 47 pequenos Phyllostomideos de Minas Gerais, apenas 2 estavam infectados, $C$. perspicillata e $L$. ecaudata, sendo negativos os da especie G. soricina. Estas tres especies parecem viver em conjunto, ou pelo menos acoitam-se em logares proximos, de preferencia casas velhas abandonadas, porões e forros escuros, boeiros. Em 1935 enconIrámos um unico C. perspicillala infeclado em B. Constant, contra 20 negativos (Dias, 1936).

No Distrito Federal achámos tambem diversos $L$. ecaudata e $C$. perspicillata parasitados, porém não apurámos exatamente a percentagem de infecção. E' esta a primeira vês que se assinala o parasitismo natural da especie $L$. ecaudata por Schizotrypanum.

$\mathrm{E}^{\prime}$ interessante notar que dentre os morcegos negativos de $\mathrm{B}$. Constant e de $\mathbf{T}$. Soares (v. relação acima), ha tres especies que foram assinaladas com hemoflagelados em outras regiões: Vampirops linealus, na Venezuela (Iturbe \& Gonzalez, 1916); Artibeus jamaicensis jamaicensis, no Panamá (Clark \& Dunn, 1932); Myotis nigricans, no Brasil (Dias, 1933, 1934), na Argentina (Romaña, 1936) e na Venezuela (Pifano, 1939). A sub-especie Glossophaga soricina leachii é um outro depositario panamense conhecido (Clark \& Dunn, 1932).

No sangue de $L$. ecaudata e de C. perspicillata do Rio de Janeiro, encontrámos, além de um Schizotrypanum, um grande tripanosoma do grupo megadermae (Dias, 1936) muito parecido com o Trypanosoma 
heybergi Rodhain (1923) do Nycteris hispidus do Congo Belga. Este parasito será estudado em outro trabalho. C. perspicillata tambem se mostrou infectada pela Bartonella rocha-limai Faria \& Pinto, 1926. ecaudata.

Passemos ao estudo do Schizotrypanum de C. perspicillala e $L$.

\section{MORFOLOGIA DO PARASITO NO SANGUE}

O parasito apresenta morfologia identica no sangue periférico de ambas especies de morcegos, tanto nos de Minas como nos do Distrito Federal. O tripanosoma é de pequenas dimensões e dotado de blefaroplasto não muito volumoso, localisado sobre o extremo posterior ou em posição sub-terminal. O corpo é geralmente incurvado em fórma de C, sendo mais espessado na parte postero-nuclear. O nucleo, ovoide ou esférico, fica bem próximo á extremidade flagelada. Flagelo livre curto, membrana ondulante estreita, quasi virtual. Protoplasma algumas vêses finamente granuloso. Em alguns individuos nota-se aparente ausencia de blefaroplasto.

Do sangue de C. perspicillata de B. Constant, em esfregaços fixados pelo alcool absoluto e corados pelo Giemsa, foram medidos 26 tripanosomas com curvimetro, após marcação á camara clara, obtendo-se as médias abaixo:

Extremidade posterior ao meio do nucleo

$6,8 \quad \mu$

Meio do nucleo á extremidade anterior

$2,6 \quad \mu$

Flagelo livre

$5,2 \mu$

Comprimento total

14,6

\section{FÓRMAS DE MULTIPLICAÇ̃̃OO}

No sangue dos morcegos não foram observados tripanosomas em via de divisão.

Nos cortes de coração de um L. ecaudata (fig. 2) foi encontrado um aglomerado parasitario composto por numerosos elementos em fórma de leishmania, aparentemente situado no interior da fibra muscular. O exame de oulros orgãos resultou negativo, assim como a pesquisa nos orgãos de um C. perspicillata.

\section{XENODIAGNOSTICO}

Um L. ecaudata de B. Constant naturalmente infectado foi sugado por diversos barbeiros normais, cuja observação foi a seguinte: 
24-3-940: Data da sucção, por varios T. infestans e $R$. prolixus.

20-4-940: Foram dissecados e examinados $3 R$. prolixus e 2 T. infestans, com resultado negativo. Em esfregaços corados de estomago de $T$. infestans não foram encontrados parasitos.

29-4-940: Dissecado e examinado 1 T. infestans, negativo.

6-5-940: Sacrificados 1 T. infestans e 1 R. prolixus, negativos.

O xenodiagnostico foi portanto negativo em 4 T. infestans e 4 R. prolixus, não obstante ter sido feito em morcego com tripanosomas não muito raros no sangue, ao exame a fresco.

Um C. perspicillata de B. Constant foi tambem sugado por $1 T$. infestans e $2 P$. megistus normais, os quais não mostraram flagelados no intestino, ao serem examinados dias mais tarde, apezar do morcego ter mostrado varios parasitos no sangue, a fresco, no dia em que foi sugado.

Em 1935 obtiveramos identico resultado, em morcego da mesma especie e da mesma região que o anterior, o qual foi sugado por $3 P$. megistus que estavam negativos ao fim de 78, 98 e 147 dias (Dias, 1936, p. 36).

No quadro abaixo estão reunidos os resultados dos xenos por nós realisados nestas duas especies de quirópteros, os quais são sugestivos da incapacidade das fórmas sanguicolas do seu Schizotrypanum se desenvolverem nas especies de reduvideos experimentadas.

XENODIAGNOSTICOS PRATICADOS EM L. ECAUD.ITA E C. PERSPICILLATA INFECTADOS (BENJAMIN CONSTANT)

MORCEGO

Lonchoglossa ecuudata

Lonchoglossa ecaudata

Carollia perspicillata

Carollia perspicillata

Carollia perspicillata

\section{BARBEIRO}

$+T$. infestans

4 R. prolixus

3 P. megistus

2 P. megistus

$1 T$. infestans
RESULTADO

Negativo

Negativo

Negativo

Negativo

Negativo

\section{INOCULAÇÃO A ANIMAIS}

O Schizotrypanum em estudo não se mostrou transmissivel a animais de laboratorio. Já em 1935 obtiveramos inoculações negalivas em 2 camondongos, inoculados com sangue de C. perspicillata de B. Constant (Dias, 1936). Recentemente, injetámos sangue do mesmo morcego e do mesmo local em 1 cobaia e 3 camondongos, que não só ficaram nega- 
livos como mostraram-se depois sensiveis á infecção por outras amostras de Schizotrypanum.

Eis a observação dos animais, que foram todos inoculados com sangue de uma Carollia no dia 26-3-40:

Cobaia 758-Até o dia 4-6-40 teve 20 exames a fresco negativos. Foi então reinoculada, com sangue do camondongo 814, infectado com a amostra phyllostomae: teve exame positivo a 21-6-40, em seguida a mais 4 exames negativos. Chegou a ter infecção muito intensa.

Camondongo 761 - Como o animal anterior, teve 20 exames negativos até 4-6-40. Reinoculado com a amostra phyllostomae, apresentou tripanosomas no sangue tambem no dia 21-6-40. Infecção prolongada.

Camondongo 762-Após 12 exames negativos até 8-5-40, foi inoculado com a amostra phyllostomae (sangue dos camondongos 769 e 770); continuou negativo até 24-5-40, aparecendo tripanosomas ao exame de 28-5-40. Morreu positivo em 2-6-40.

Camondongo 760-Até 29-4-40 teve 9 exames negativos. Reinoculado (S. cruzi, amostra vickersae) neste dia, teve exame positivo a 7-5-40, tendo sido sacrificado, ainda infectado, a 18-6-40.

\section{CULTURA}

O Schizotrypanum de Carollia perspicillata é cultivavel em meio de Noguchi. Isolámos o parasito anteriormente (Dias, 1936) de morcego de B. Constant, e de novo, no Distrito Federal. Nas culturas desenvolvidas encontram-se parasitos critidiomorficos e tripaniformes. Ainda está por ser feito o estudo comparativo das fórmas culturais deste flagelado com as de outros do mesmo genero.

\section{DISCUSS̃̃O}

Segundo as investigações levadas a cabo no presente trabalho, os morcegos Carollia perspicillata e Lonchoglossa ecaudata de Minas Gerais e do Distrito Federal apresentam-se naturalmente infectados por um flagelado do genero Schizotrypanum. Trata-se de um flagelado não transmissivel a animais de laboratorio (cobaia, camondongo), cultivavel in vitro, que não se mostrou capaz de evoluir em diversos reduvideos (Pantrongylus megistus, Triatoma infestans, Rhodnius prolixus) transmissores do Schizotrypanum cruzi.

No sangue de ambos os hospedeiros o parasito caracterisa-se morlologicamente: $10^{\circ}$ pelo pequeno comprimento total; $2{ }^{\circ}$ pela situação do 
nucleo, muito proximo á extremidade anterior do corpo. As formas de tripanosoma são muito semelhantes ás do $S$. vespertilionis (Battaglia), conforme o demonstram as ilustrações do presente trabalho (figs. 1 e 2). Quasi todos os autores que estudaram os tripanosomas do sangue de morcegos europeus ou africanos ( $S$. vespertilionis) chamam a atenção para a colocação do nucleo "mais proximo" ou "muito proximo " ao extremo anterior do corpo do protozoario: Battaglia (1904), Sergent (1905), Kisskalt (1906), Nicolle \& Comte (1906), Bettencourt \& França (1907) Gonder (1910), Coles (1914), Chatton \& Courrier (1921), assinalando lambem, alguns deles, o pequeno tamanho do parasilo. Bettencourt \& França (1907) dão uma perfeita descrição do $S$. vespertilionis dos quirópteros de Portugal, identificando-o ao Trypanosoma nicolleorum, ao $T$. dionisii e aos tripanosomas estudados por Petrie (1905) e Kisskalı (1906), após terem examinado o material original destes pesquizadores. Assinalam que o nucleo quasi sempre está a apenas $1,5 "$ do ponto de onde emerge o flagelo livre, isto é, muito anteriormente situado. Gonder (1910, p. 296) chega a afirmar: "Die Trypanosomen aus dem Blute der Fledermäuse sind... vor allem durch die Lage ihrer Kerne charakterisiert; der Kern liegt weit im Vorderende, der Blepharoplast ganz im Hinterende ».

Com respeito a outros flagelados de morcegos americanos, o Schizotrypanum em estudo parece assemelhar-se sómente ao do Myotis nigricans, que, além de ter o nucleo situado muito para a frente, não se mostrou nem inoculavel a animais nem transmissivel pelo $T$. infestans. Os dados disponiveis sobre a morfologia e a biologia da maioria dos parasitos até agora encontrados, entretanto, são ainda muito incompletos para que possamos identifica-los ou diferença-los uns dos outros. No quadro n. ${ }^{\circ} 2$ colocamos os resultados até agora conhecidos de inoculações em animais, xenodiagnostico e exame histologico em quirópteros do Novo Mundo, chamando especial atenção para os resultados negalivos de xenodiagnostico, que são talvez indicação de que possivelmente se trata, nesses casos, de parasitos do tipo "vespertilionis". Referimo-nos aqui a este tipo no sentido estricto definido neste trabalho (forma sanguicola pequena, de nucleo bem anterior), e não ao tipo vespertilionis na accepção ampla que demos em trabalho anterior (Dias, 1936), que abrangia todos os parasitos do genero Schizotrypanum.

De acordo com os resultados de um estudo biometrico de varios parasitos deste genero (Dias \& Freitas), foram verificadas diferenças morfologicas significativas entre diversas amostras de distinta procedencia, diferenças essas que dizem respeito sobretudo á posição do nucleo. No quadro n. ${ }^{0} 1$ comparamos as medidas médias e extremas que obti- 
Quadro 1

Medidas obtidas de tripanosomas do sangue periferico, desenhado á camara clara e metidos com curvimetro (alcool absoluto Giemsa).

\begin{tabular}{|c|c|c|c|c|c|c|c|c|c|c|c|c|c|c|}
\hline \multirow[t]{2}{*}{ A MOSTRA } & \multicolumn{3}{|c|}{ P. N. } & \multicolumn{3}{|c|}{ N. A. } & \multicolumn{3}{|c|}{ Flagelo } & \multicolumn{3}{|c|}{ Total } & \multirow{2}{*}{$\frac{\text { PN/NA }}{\text { Md. }}$} & \multirow{2}{*}{ 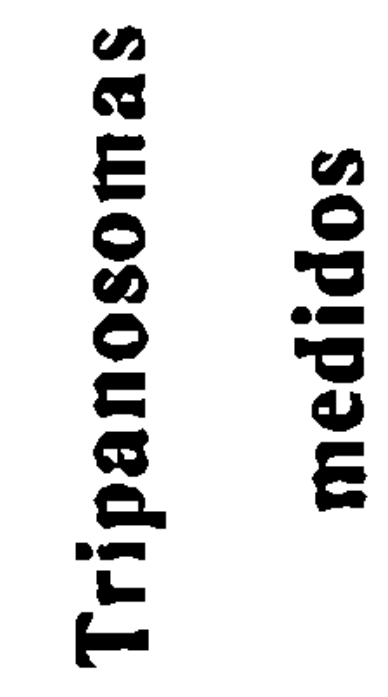 } \\
\hline & $\mathrm{Mn}$. & $M x$ & $M d$. & $M n$. & Mx. & Md. & $M n$. & $M x$. & Md. & Mn. & $M x$ & Md. & & \\
\hline Vespertilionis & 5,4 & 8,5 & 6,8 & 1,8 & 4,2 & 2,6 & 3.6 & 6,7 & 5,2 & 11,4 & 17.0 & 14,6 & 2,6 & 26 \\
\hline Cruzi humano & 4,9 & 9,1 & 6,7 & 2,1 & 5,9 & 4,0 & 5,2 & 8,4 & 6,7 & 13,6 & 20,6 & 17,4 & 1,6 & 100 \\
\hline Phyllostomae & 4,8 & 9,1 & 7,1 & 3,0 & 6,7 & 5,0 & 5,4 & 9,7 & 7,8 & 15,1 & 23,6 & 20,0 & 1,4 & 100 \\
\hline Hastatus & 4,5 & 8,7 & 6,4 & 4,2 & 8,0 & 6,2 & 5,4 & 10,5 & 7,7 & 16,2 & 25,1 & 20,4 & 1,0 & 100 \\
\hline
\end{tabular}

P. N. - Distancia do extremo posterior ao meio do nucleo.

N. A. - Distancia do meio do nucleo ao extremo anterior do corpo.

PN/NA - Relação que exprime a posição do nucleo. 
vemos de 26 tripanosomas do sangue de Carollia perspicillata de Minas Gerais (amostra "vespertilionis"), com as obtidas de 100 tripanosomas de cada uma das amostras "cruzi ", "phyllostomae " e "hastalus ". As duas ultimas são de morcegos, respectivamente de Carollia perspicillata da Venezuela e de Phyllostomus hastatus do Brasil. Denominamos "phyllostomae a uma delas por corresponder, pela morfologia das formas sanguicolas, ao Trypanosoma phyllostomae Cartaya 1910, descrito no morcego $C$. perspicillata de Cuba. Ao descrever este parasito o autor assinalou que "El (tripanosoma) que hemos encontrado en el A. perspicillatus y que provisionalmente nombraremos Trypanosoma phyllostomae no parece corresponder en sus medidas á ninguno de los anteriores ", isto é, ao $T$. vespetilionis e outros até então descritos em sangue de morcegos.

A amostra que medimos de C. perspicillata da Venezuela, e sobre a qual Dias \& Pifano estão por publicar um trabalho, é um Schizotrypanum patogenico para os animais de laboratorio e transmissivel por barbeiros e outros hematófagos. Biometricamente ela se distingue tambem do Schizotrypanum cruzi humano e do Schizotrypanum do morcego Phyllostomus hastatus.

As tres amostras (cruzi, phyllostomae e hastatus) diferem morfologicamente da "vespertilionis" pelas suas dimensões maiores e pela situação do nucleo mais medianamente, e biologicamente por evoluirem facilmente em reduvideos. Além disto, as amostras "phyllostomae " $\mathrm{e}$ "cruzi " são inoculaveis a diversos animais. A "hastatus", que biometricamente é a que mais se distancia da "vespertilionis", tem em comum com esta a não transmissibilidade aos animais de experiencia, só excepcionalmente conseguida (Dias, 1936). Com toda a razão afirmou Rodhain (1939) não ser o Schizotrypanum do Phyllostomus hastatus morfologicamente identico ao $S$. vespertilionis dos morcegos europeus, que ele teve oportunidade de estudar.

Do ponto de vista morfologico o Schizotrypanum cruzi ocupa logar intermediario entre as amostras phyllostomae e vespertilionis, tanto pelo lamanho como pela posição do nucleo; e a phyllostomae é intermediaria entre a cruzi e a hastatus (v. quadro 1).

Os 4 tipos de Schizotrypanum em consideração, não obstante as diferenças assinaladas, têm caracteres morfologicos e biologicos comuns, lais como: aspéto geral dos tripanosomas, com blefaroplasto mais ou menos volumoso sub-terminal ou terminal; indivisibilidade das formas sanguicolas adultas; proliferação nos tecidos sob a forma de leishmania; cultivabilidade in vitro; evolução em "posterior station " no transmissor (nem sempre conhecido). 
Com relação ás formas proliferalivas, refiramos que no Pipistrellus pipistrellus da Europa elas foram assinaladas primeiramente por Coles (1915) como "immalure trypanosomes", sendo depois referidos os "quistos critidianos" de Chatton \& Courrier (1921) e finalmente encontradas leishmanias por Franchini (1922).

Pelo quadro n.o 2 podemos observar que em muitos morcegos americanos infectados até agora não foram encontrados os elementos de multiplicação.

O S. vespertilionis dos morcegos europeus e africanos tem em comum com o parasito que estudamos, além da morfologia dos tripanosomas sanguicolas, a não infectividade para animais, que foi comprovada no primeiro pela grande maioria dos autores. Com respeito ao xenodiagnostico, não sabemos de ensaios praticados com barbeiros em morcegos de outros continentes; entretanlo Rodhain (1939), trabalhando com culturas do $S$. vespertilionis, que fazia ingerir por hemalófagos, observou a prolongada sobrevivencia dos flagelados no organismo de Panstrongylus megistus, Cimex lectularius e Ornithodoros monbata.

\section{RESUMO E CONCLUSÕES}

1) Fica assinalada a infecção natural dos Phyllostomideos Lonchoglossa ecaudata e Carollia perspicillata no Fstado de Minas Gerais e na cidade do Rio de Janciro, Brasil, por um Schizotrypanum.

2) Em ambos os morcegos as formas de tripanosoma apresentam morfologia identica, caracterisada principalmente por suas pequenas dimensões $\left(15^{\mu}\right)$ e pelo nucleo localisado muito proximo ao extremo anterior do corpo.

3) Tais caracteristicas morfologicas são muito visinhas, sinão identicas, ás do Schizotrypanum vespertilionis (Battaglia), e distinguem o parasilo em estudo do Schizotrypanum cruzi humano. de um Schizotrypanum do morcego Carollia perspicillata da Venezuela (=T. phyllostomae Cartaya?) e do Schizotrypanum do morcego Phyllostomus hastatus do Brasil.

4) Em córtes de coração de Lonchoglossa ecaudala foram encontrados parasitos com a morfologia de leishmania.

5) Xenodiagnosticos realisados com Panstrongylus megistus, Tria- 
Quadro 2

Dados biologicos existentes sobre hemo flagelados dos morcegos do Novo Mundo, relativos a inoculação em animal,

xenodiagnostico e formas de multiplicação nos tecidos.

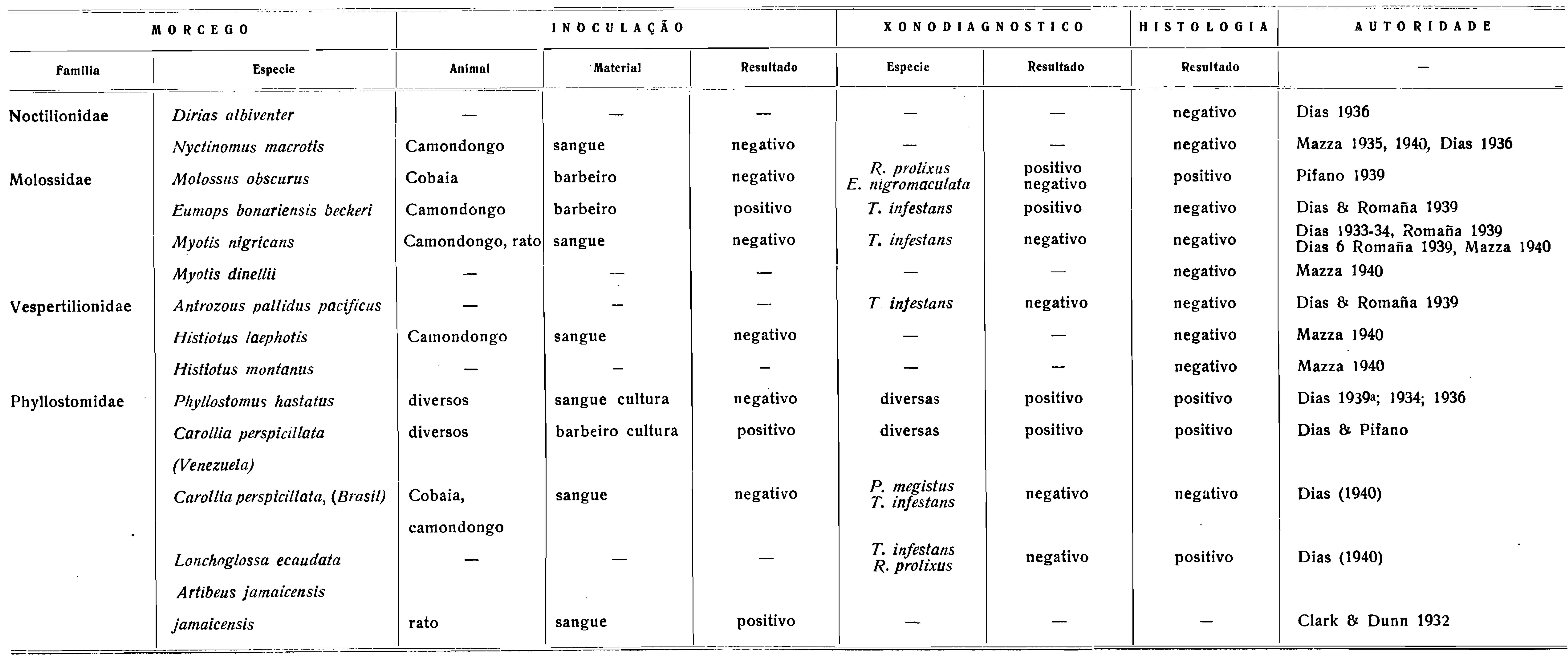


toma infestans e Rhodnius prolixus, em morcegos com tripanosomas no sangue ao cxame a fresco, resultaram negativos.

6) Em ambas as especies de morcegos, no Rio de Janeiro, foi encontrado tambem um grande tripanosoma do grupo megadermae, parecido com o Trypanosoma heybergi Rodhain do morcego africano Nycteris hispidus. Em C. perspicillata da mesma cidade foi verificada tambem a infecção pela Barłonella rocha-limai Faria \& Pinto, e tambem a presença de microfilarias no sangue. No rim de C. perspicillata de B. Constant encontrámos fórmas esquisogonicas de um protozoario.

\section{AGRADECIMENTOS}

Expressamos nosso reconhecimento aos Drs. Oliverio M. de Oliveira Pinlo e Carlos Vieira, do Museu Paulista, pela determinação dos uossos morcegos. E aos Srs. Dr. Romeu Ramos, Luiz Monteiro, Boanerges Tosles e Carlos Ramos, pelo enlusiasmo com que nos auxiliaram na obtenção do material de estudo.

\section{SUMMARY}

1) Infection by a flagellate of the genus Schizotrypanum has been delected in the bats Lonchoglossa ecaudata and Carollia perspicillata from Minas Geraes and Rio de Janeiro, D. F.

2) The blood trypanosome forms are characterized by its short length $\left(15^{\prime \prime}\right)$ and by its nucleus situated very near the anterior end of the body.

3) Morphologically the parasite is closely related, if not identical to Schizotrypanum vespertilionis (Battaglia), and it differs from Schizotrypanum cruzi Chagas, from a Schizotrypanum of Carollia perspicillata from Venezucla (= Trypanosoma phylloslomae Cartaya?) and from the Schizotrypanum of Phyllostomus hastatus.

4) Leishmania bodies have been found in scclions of heart of Lonchoglossa ecaudata.

6) Xenodiagnostics perfomed on infected bats of bolh species proved negative with several species of bugs (Panslrongylus megistus, Triatoma infestans, Rhodnius prolixus). 
6) A large Trypanosome similar to T. heybergi Rodhain has been encountered in the blood of $C$. perspicillata and $L$. ecaudata from Rio de Janeiro, D. F. C. perspicillata from this city also filaria. Schizogonic forms of a prolozoa have been found in the kidneis of $C$. perspicillala from B. Constant.

\section{BIBLIOGRAFIA}

Battaglia, M.

1904. Ann. Med. Navarra, 7 (2): 254. Citado por Bettencourt \& França, 1907 .

Bettencourt, A. \& França, C.

1907. Arch. R. Inst. Bact. Camara Pestana, 1 : 187-194.

Cattaya, J. T.

1910. Sanidad \& Beneficencia (Habana), $3: 507$.

Cinttion, E. \& Courrier, R.

1921. C. R. Soc. Biol., $84: 943$; C. R. Acad. Sc., $172: 1954$.

Cluark, H. C. \& Dunn, L. H.

1932. Am. Jl. Trop. Med., $12: 49$.

Coles, A. C.

1915. Parasitology, $7:$ 17-61.

DiAs, E.

1933 a. Memorias do Inst. Oswaldo Cruz, 27 : 139.

$1933 b$. Tése de Doutoramento, Rio de Janeiro.

1934. Memorias do Inst. Oswaldo Cruz, 28 : 1-111.

1936. IX Reunião da Soc. Argentina Pat. Regional (M. E. P. R. A.), 1 : 10-8S

Dias, E. \& Freitas, L.

Em impressão.

Dias, E. \& Pifano, C. F.

Em impressão proxima.

Dias, E. \& Romana, C.

1939. Memorias do Inst. Oswaldo Cruz, $34(4): 619-625$.

Faria, J. G. \& Pinto, C.

1926. Boletim Biol., São Paulo (2) : 21-23.

Franchini, G.

1922. Bull. Soc. Pat. Ex., $14: 542$. 
Gonder, R.

1910. Centr. f. Bakt., Orig., $53: 293$.

Ituribe, J. \& Gonzalez, E.

1916. Ref. Trop. Dis. Bull., 9 : 342.

KisSKaLt, K.

1906. Centr. f. Bakt., Orig., $40: 213$.

MazzA, S.

1935. Publ. n.o 22, M. E. P. R. A.

1936. IX Reunião da Soc. Argentina Pat. Regional, $1: 172$.

1940. Publ. n.o 45, M. E. P. R. A. : 119-134.

Nicolle, C. \& Comte, C.

1906. C. R. Soc. Biol., $58: 736$.

Petrie, G. F.

1905. Jour. Hyg., 5 : 191.

Pifano, F.

1939. Policlinica Caracas, n.o 46, Junho.

1939. Gaceta Med. Caracas, 47 (22) : 435-436.

Rodhain, J.

1923. Bull. Soc. Pat. Ex., 16 : 659.

1939. C. R. Soc. Biol., 131 (19) : 814-818.

Romana, C.

1936. IX Reunião Soc. Arg. Pat. Reg., 1 : 232-234.

Sergent, E. \& Sergent, E.

1905. C. R. Soc. Biol., $58: 53$. 


\section{Estampa 1}

Fig. 1 - Tripanosoma do sangue periférico de Carollia perspicillata de Benjamin Constant. Alcool absoluto, Giemsa. 1500 vêses.

[rig. 2 - Tripanosoma vespertilionis no sangue de morcegos europeus, segundo Bettencourt \& França (1907, estampa 3, fig. 3) e Coles (1915, plate II: figs. 8, 9 e 11). Vêem-se os "immature trypanosomes» de Coles.

Fig. 3-Fórmas de leishmania do coração de Lonchoglossa ecaudala de Benjamin Constant. Formol, Hematoxilina-Eosina. 1500 vêses.

Foto M. Cezar

S. E. G. E., I. O. C. 
35 (2), SET., 1940
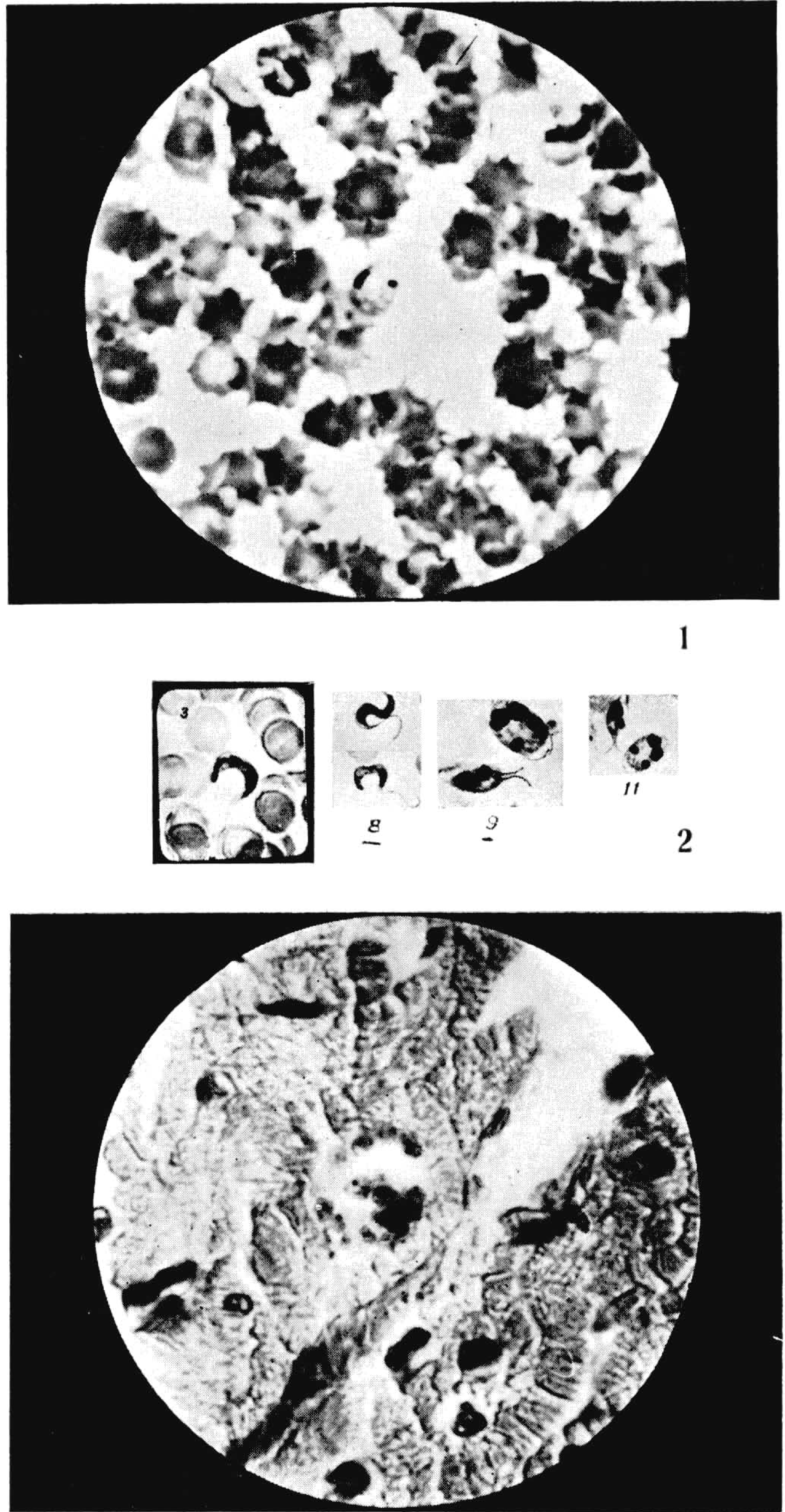

Nota: 0 aumento das figuras 1 e 3 é de 1240 vezes, e não de 1500 conforme consta na explicação da estampa. 